\title{
Fidelity of quantum teleportation through noisy channels
}

\author{
Sangchul Oh,* Soonchil Lee, ${ }^{\dagger}$ and Hai-woong Lee \\ Department of Physics, Korea Advanced Institute of Science and Technology, Daejon 305-701, Korea \\ (Received 25 January 2002; revised manuscript received 21 May 2002; published 27 August 2002)
}

\begin{abstract}
We investigate quantum teleportation through noisy quantum channels by solving analytically and numerically a master equation in the Lindblad form. We calculate the fidelity as a function of decoherence rates and angles of a state to be teleported. It is found that the average fidelity and the range of states to be accurately teleported depend on types of noises acting on quantum channels. If the quantum channels are subject to isotropic noise, the average fidelity decays to $1 / 2$, which is smaller than the best possible value of $2 / 3$ obtained only by the classical communication. On the other hand, if the noisy quantum channel is modeled by a single Lindblad operator, the average fidelity is always greater than $2 / 3$.
\end{abstract}

DOI: 10.1103/PhysRevA.66.022316

PACS number(s): 03.67.Hk, 03.65.Yz, 03.67.Lx, 05.40.Ca

Quantum teleportation $[1,2]$ is a process by which a sender, called Alice, transmits an unknown quantum state to a remote recipient, called Bob, via dual classical and quantum channels. Here a pair of maximally entangled particles, forming a quantum channel, should be used for the perfect quantum teleportation. However, while being distributed and kept by Alice and Bob, an entangled state may lose its coherence and become a mixed state due to the interaction with its environment.

Bennett et al. [1] noted that the quantum channel that is less entangled reduces the fidelity of teleportation, and/or the range of states that can be accurately teleported. Popescu [3] investigated the relations among teleportation, Bell's inequalities, and nonlocality. It was demonstrated that there are mixed states that do not violate any Bell-type inequality, but still can be used for teleportation. Horodecki et al. [4] showed that any two mixed spin- $\frac{1}{2}$ states that violate the Bell-CHSH inequality are useful for teleportation. Also Horodecki et al. [6] proved the relation between the optimal fidelity of teleportation and the maximal singlet fraction of the quantum channel. Banaszek [7] investigated the fidelity of quantum teleportation using nonmaximally entangled states. Ishizaka [8] studied the quantum channel subject to local interaction with two-level environment. Although the studies cited above reveal the important relations between the degree of entanglement of the quantum channel and quantum teleportation, there seem to be little studies on the direct connection between the quantum teleportation and decoherence rates. Thus it might be interesting to know how the type and strength of noise acting on quantum channels affect the fidelity of quantum teleportation.

In this paper, we investigate quantum teleportation through noisy channels by solving analytically and numerically a master equation in the Lindblad form. We obtain the fidelity of quantum teleportation as a function of decoherence time and angles of an unknown state to be teleported. Thus we explicitly demonstrate Bennett et al.'s argument that noisy quantum channels reduce the range of states to be

\footnotetext{
*Electronic address: scoh@mrm.kaist.ac.kr

${ }^{\dagger}$ Electronic address: sclee@mail.kaist.ac.kr

¥Electronic address: hwlee@laputa.kaist.ac.kr
}

accurately teleported. We also examine the characteristic dependence of the average fidelity on types of noises acting on qubits at each stage of the teleportation.

Let us consider quantum teleportation through noisy channels as illustrated in Fig. 1. The top two qubits are taken by Alice and the bottom qubit is kept by Bob. Here, measurements are performed at the end of the circuit for computational convenience. Classical conditional operations can be replaced with corresponding quantum conditional operations [9]. Decoherence of an open quantum system is due to the interaction with its environment. Under the assumption of Markov and Born approximations and after tracing out the environmental degrees of freedom, the dynamics of an open quantum system is described by a master equation for the density operator of the quantum system alone, $\rho(t)$, in the Lindblad form $[10,11]$

$$
\frac{\partial \rho}{\partial t}=-\frac{i}{\hbar}\left[H_{S}, \rho\right]+\sum_{i, \alpha}\left(L_{i, \alpha} \rho L_{i, \alpha}^{\dagger}-\frac{1}{2}\left\{L_{i, \alpha}^{\dagger} L_{i, \alpha}, \rho\right\}\right),
$$

where the Lindblad operator $L_{i, \alpha}=\sqrt{\kappa_{i, \alpha}(t)} \sigma_{\alpha}^{(i)}$ acts on the $i$ th qubit and describes decoherence. Throughout this paper, $\sigma_{\alpha}^{(i)}$ denotes the Pauli spin matrix of the $i$ th qubit with $\alpha$ $=x, y, z$. The decoherence time is approximately given by $1 / \kappa_{i, \alpha}$. We could control noise by switching $\kappa_{i, \alpha}(t)$ on and off. We take the Hamiltonian of a qubit system as an ideal model of a quantum computer, which is given by [12]

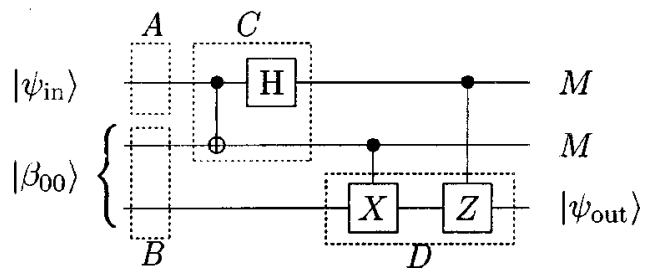

FIG. 1. A circuit for quantum teleportation through noisy channels. The two top lines belong to Alice, while the bottom one belongs to Bob. $M$ represents measurement. The dotted boxes $A, B, C$, and $D$ denote noisy channels. Time advances from left to right. During the time interval corresponding to the width of the dotted box, the Lindblad operator is turned on. 


$$
H_{S}(t)=-\frac{1}{2} \sum_{i=1}^{N} \mathbf{B}^{(i)}(t) \cdot \boldsymbol{\sigma}^{(i)}-\sum_{i \neq j} J_{i j}(t) \sigma_{+}^{(i)} \sigma_{-}^{(j)},
$$

where $\boldsymbol{\sigma}^{(i)}=\left(\sigma_{x}^{(i)}, \sigma_{y}^{(i)}, \sigma_{z}^{(i)}\right)$ and $\sigma_{ \pm}^{(i)}=\frac{1}{2}\left(\sigma_{x}^{(i)} \pm i \sigma_{y}^{(i)}\right)$. In solid-state qubits, various types of couplings between qubits $i$ and $j$ are possible such as the $X-Y$ coupling given above, the Heisenberg coupling, and the Ising coupling $J_{i j} \sigma_{z}^{(i)} \sigma_{z}^{(j)}$ in nuclear magnetic resonance. The various quantum gates in Fig. 1 could be implemented by a sequence of pulses, i.e., by turning on and off $\mathbf{B}^{(i)}(t)$ and $J_{i j}(t)$. We develop the simulation code that solves Eq. (1), the set of differential equations for the density matrix $\rho_{m n}(t)$, based on the RungeKutta method [13]. Equation (1) shows $\operatorname{Tr} \rho(t)=1$ at all times.

An unknown state to be teleported can be written as $\left|\psi_{\text {in }}\right\rangle=\alpha|0\rangle+\beta|1\rangle$ with $|\alpha|^{2}+|\beta|^{2}=1$. It is convenient to rewrite $\left|\psi_{\text {in }}\right\rangle$ as a Bloch vector on a Bloch sphere,

$$
\left|\psi_{\text {in }}\right\rangle=\cos \left(\frac{\theta}{2}\right) e^{i \phi / 2}|0\rangle+\sin \left(\frac{\theta}{2}\right) e^{-i \phi / 2}|1\rangle,
$$

where $\theta$ and $\phi$ are the polar and azimuthal angles, respectively. The maximally entangled state of two spin- $\frac{1}{2}$ particles shared and kept by Alice and Bob is given by

$$
\left|\beta_{00}\right\rangle \equiv \frac{1}{\sqrt{2}}(|00\rangle+|11\rangle) .
$$

The input state of the quantum teleportation circuit in Fig. 1 is the product state of $\left|\psi_{\text {in }}\right\rangle$ and $\left|\beta_{00}\right\rangle$. After the implementation of the quantum circuit of Fig. 1 and the measurement of the top two qubits, Bob gets the teleported state $\left|\psi_{\text {out }}\right\rangle$. It is useful to describe the teleportation in terms of density operators

$$
\rho_{\text {out }}=\operatorname{Tr}_{1,2}\left\{U_{\text {tel }} \rho_{\text {in }} \otimes \rho_{\text {en }} U_{\text {tel }}^{\dagger}\right\},
$$

where $\rho_{\text {in }}=\left|\psi_{\text {in }}\right\rangle\left\langle\psi_{\text {in }}\left|, \rho_{\text {en }}=\right| \beta_{00}\right\rangle\left\langle\beta_{00}\right|$, and $\operatorname{Tr}_{1,2}$ is a partial trace over qubits 1 and 2 . The unitary operator $U_{\text {tel }}$ is implemented by the teleportation circuit as shown in Fig. 1. If the teleportation is ideal, the density-matrix teleported $\rho_{\text {out }}$ is identical to $\rho_{\text {in }}$ up to the normalization factor.

As illustrated as dotted boxes in Fig. 1, we consider four different noisy channels, $A, B, C$, and $D$. In case $A$ an unknown state $\left|\psi_{\text {in }}\right\rangle$ loses its coherence and becomes a mixed state before it is teleported. In case $B$ an entangled pair, forming a quantum channel, becomes noisy while being shared and kept by Alice and Bob. In cases $C$ and $D$, while Alice and Bob perform the Bell measurement and the unitary operation, respectively, noise may set in. For cases $A$ and $B$ we obtain both analytic and numerical solutions of Eq. (1), while in cases $C$ and $D$ the numerical solutions of Eq. (1) are obtained. For our numerical calculation, $\kappa_{i, \alpha}(t)$ is turned on for the time interval $\tau$ corresponding to the width of each dotted box in Fig. 1.

The properties of quantum teleportation through noisy quantum channels are quantified by the fidelity that measures the overlap between a state $\left|\psi_{\text {in }}\right\rangle$ to be teleported and the density operator $\rho_{\text {out }}$ for a teleported state,

$$
F(\theta, \phi)=\left\langle\psi_{\text {in }}\left|\rho_{\text {out }}\right| \psi_{\text {in }}\right\rangle .
$$

Here the fidelity $F(\theta, \phi)$ depends on an input state as well as the type of noise acting on qubits. We calculate $F(\theta, \phi)$ and determine the range of states $\left|\psi_{\text {in }}\right\rangle$ which can be accurately teleported. Since in general a state to be teleported is unknown, it is more useful to calculate the average fidelity given by

$$
F_{\mathrm{av}}=\frac{1}{4 \pi} \int_{0}^{\pi} d \theta \int_{0}^{2 \pi} d \phi F(\theta, \phi) \sin \theta
$$

where $4 \pi$ is the solid angle.

Case A: States to be teleported are mixed. Alice is not able to know or copy the state to be teleported without disturbing it. So it may be pure or mixed. As Bennett et al. [1] noted, the linear property of quantum teleportation enables one to teleport not only a pure state but also a mixed state. The quantum operation $\mathcal{E}$ transforms a pure state $\rho_{\text {in }}$ $=\left|\psi_{\text {in }}\right\rangle\left\langle\psi_{\text {in }}\right|$ to a mixed state $\mathcal{E}\left(\rho_{\text {in }}\right)$. The time evolution of pure states to mixed states is described by Eq. (1) (see Ref. [9] for the connection between the two approaches). From Eq. (5), quantum teleportation of mixed states reads

$$
\mathcal{E}\left(\rho_{\text {out }}\right)=\operatorname{Tr}_{1,2}\left\{U_{\text {tel }} \mathcal{E}\left(\rho_{\text {in }}\right) \otimes \rho_{\text {en }} U_{\text {tel }}^{\dagger}\right\} .
$$

The decoherence of the state to be teleported, $\mathcal{E}\left(\rho_{\text {in }}\right)$, is transferred to the state teleported, $\mathcal{E}\left(\rho_{\text {out }}\right)$. For various types of noises, we obtain both analytic and numerical solutions of Eq. (1) and calculate the fidelity.

Suppose a state to be teleported is subject to the noise $L_{1, z}$. It is easy to find the analytic solution of Eq. (1) when $H_{S}(t)=0$. We obtain the mixed state to be teleported, $\mathcal{E}\left(\rho_{\text {in }}\right)$, as $\quad \rho^{(00)}(t)=\rho_{\text {in }}^{(00)}(0), \quad \rho^{(11)}(t)=\rho_{\text {in }}^{(11)}(0), \quad$ and $\quad \rho^{(01)}(t)$ $=\rho_{\text {in }}^{(01)}(0) \exp (-2 \kappa t)$. Then from Eqs. (8) and (6), the fidelity can be calculated as

$$
F(\theta, \phi)=1-\frac{1}{2}\left(1-e^{-2 \kappa \tau}\right) \sin ^{2} \theta .
$$

If $2 \kappa \tau \ll 1, F(\theta, \phi) \simeq 1-\kappa \tau \sin ^{2} \theta$. On the other hand, if $2 \kappa \tau \gg 1, F(\theta, \phi) \simeq \frac{1}{2}\left(1+\cos ^{2} \theta\right)$. Figure 2(a) is the plot of Eq. (9) for $2 \kappa \tau=3.0$.

Let us consider that the state $\left|\psi_{\text {in }}\right\rangle$ is subject to the noise described by $L_{1, x}$. After some calculations, we obtain the fidelity

$F(\theta, \phi)=\frac{1}{2}\left[1+\sin ^{2} \theta \cos ^{2} \phi+e^{-2 \kappa \tau}\left(\cos ^{2} \theta+\sin ^{2} \theta \sin ^{2} \phi\right)\right]$.

If $2 \kappa \tau \ll 1, F(\theta, \phi) \simeq 1-\kappa \tau\left(\cos ^{2} \theta+\sin ^{2} \theta \sin ^{2} \phi\right)$. In the limit of $2 \kappa \tau \gg 1$, we have $F(\theta, \phi) \simeq \frac{1}{2}\left(1+\sin ^{2} \theta \cos ^{2} \phi\right)$. The plot of Eq. (10) at $2 \kappa \tau=3.0$ is shown in Fig. 2(b).

Substituting Eq. (10) or (9) into Eq. (7), we get the average fidelity 

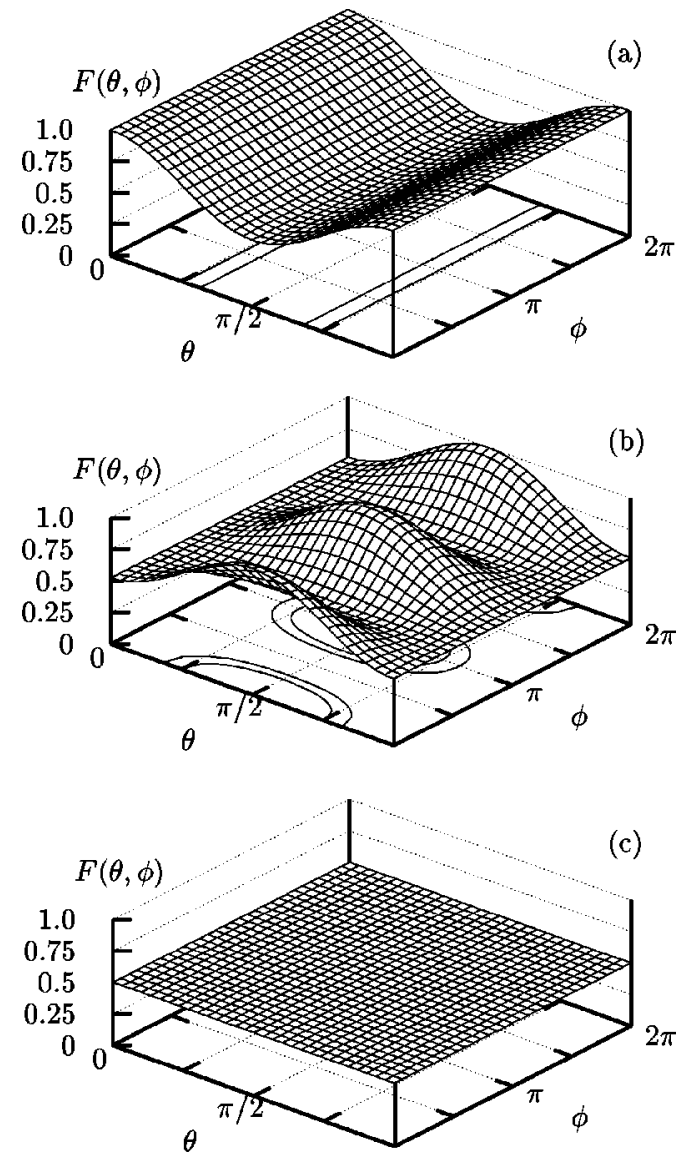

FIG. 2. Fidelity $F(\theta, \phi)$ as a function of angles $\theta$ and $\phi$ of the state to be teleported for case $A$ at $2 \kappa \tau=3.0$ and for case $B$ at $4 \kappa \tau=3$.0. For case $B$ in (a), the Lindblad operators $L_{2, z}$ and $L_{3, z}$, and in (b), $L_{2, x}$ and $L_{3, x}$ are turned on. The maximum value of the fidelity of (a) and (b) is 1 and the minimum is $1 / 2$. The contours on the $\theta-\phi$ planes in (a) and (b) join the points with the fidelities $F(\theta, \phi)=3 / 4$ and $2 / 3$, respectively. In (c), the isotropic noise is applied.

$$
F_{\mathrm{av}}(\tau)=\frac{2}{3}+\frac{1}{3} e^{-2 \kappa \tau}
$$

In Fig. 3, the solid line (denoted by case $A-1$ ) shows the plot of Eq. (11), the average fidelity as a function of $\kappa \tau$ for the noise modeled by $L_{1 x}$ or $L_{1 z}$.

Now suppose the isotropic noise $\left(L_{1 x}, L_{1 y}\right.$, and $\left.L_{1 z}\right)$ is applied to the state $\left|\psi_{\text {in }}\right\rangle$. The analytic solution of Eq. (1) gives us the fidelity written by

$$
F_{\mathrm{av}}=F(\theta, \phi)=\frac{1}{2}+\frac{1}{2} e^{-4 \kappa \tau} .
$$

If $4 \kappa \tau \ll 1, \quad F(\theta, \phi) \simeq 1-2 \kappa \tau$. For $4 \kappa \tau \gg 1$, we have $F(\theta, \phi) \simeq \frac{1}{2}$, as shown in Fig. 2(c). In Fig. 3, the dotted line (denoted by case $A-2$ ) is the plot of Eq. (12).

Case B: Quantum channels are noisy. While being distributed and stored by Alice and Bob, an entangled state of two spin- $\frac{1}{2}$ particles may be subject to noise. The dynamics of an entangled pair subject to quantum noise is described by the quantum operation $\mathcal{E}$ acting on the pure entangled state,

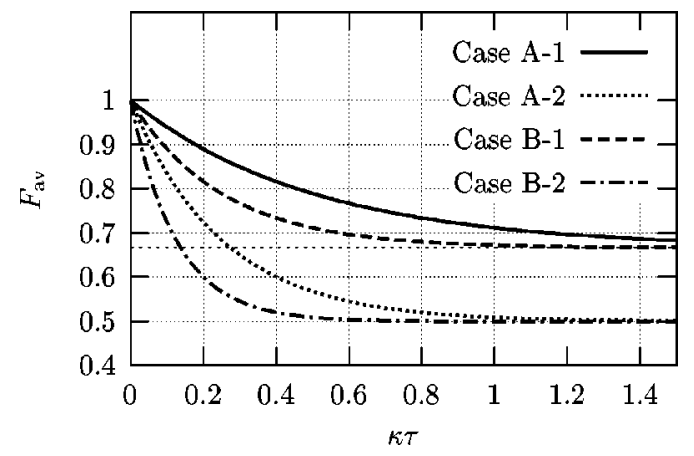

FIG. 3. Average fidelity $F_{\text {av }}$ as a function of $\kappa \tau$ for cases $A$ and $B$. The solid line (case $A-1$ ) is the plot of Eq. (11) for the noise described by $L_{1, x}$ (or by $L_{1, z}$ ). The dotted line (case $A-2$ ) is based on Eq. (12) corresponding to the isotropic noise. The dashed line (case $B-1$ ) is for Eq. (14), the noise modeled by $L_{2, x}$ and $L_{3, x}$ (or by $L_{2, z}$ and $L_{3, z}$ ). The dash-dotted line (case $B-2$ ) is the plot of Eq. (15) for the isotropic noise. The horizontal dotted line with $2 / 3$ shows the maximum fidelity obtained only by the classical communication.

$\rho_{\text {en }} \rightarrow \mathcal{E}\left(\rho_{\text {en }}\right)$, or by Eq. (1). From Eq. (5), the quantum teleportation with noisy quantum channels can be written as

$$
\mathcal{E}\left(\rho_{\text {out }}\right)=\operatorname{Tr}_{1,2}\left\{U_{\text {tel }} \rho_{\text {in }} \otimes \mathcal{E}\left(\rho_{\text {en }}\right) U_{\text {tel }}^{\dagger}\right\}
$$

We find that the quantum teleportation process transfers the decoherence of the entangled pair $\mathcal{E}\left(\rho_{\text {en }}\right)$ to that of the output state $\mathcal{E}\left(\rho_{\text {out }}\right)$. It should be noted that the quantum operation acting on the entangled pair $\mathcal{E}\left(\rho_{\text {en }}\right)$ is not only a $4 \times 4$ matrix, but effectively a $2 \times 2$ matrix. Thus overall features of case $B$ are similar to case $A$ except the decoherence rates.

Consider the quantum channel subject to the noise acting in one direction, for example, the $z$ direction. This type of noise is modeled by Lindblad operators $L_{2, z}=\sqrt{\kappa_{2, z}} \sigma_{z}^{(2)}$ and $L_{3, z}=\sqrt{\kappa_{3, z}} \sigma_{z}^{(3)}$ acting on an entangled pair, qubits 2 and 3 , respectively. Here we assume the same strength of decoherence rates, $\kappa \equiv \kappa_{2, z}=\kappa_{3, z}$. We obtain the fidelity $F(\theta, \phi)$ with the same form of Eq. (9) except the replacement of $2 \kappa \tau$ with $4 \kappa \tau$. That is, $F(\theta, \phi)=1-\frac{1}{2}[1-\exp (-4 \kappa \tau)] \sin ^{2} \theta$. For the noise described by $L_{2, x}$ and $L_{3, x}$, the fidelity $F(\theta, \phi)$ is identical to the form of Eq. (10) with exponent $4 \kappa \tau$.

Let us discuss Bennett et al.'s argument: the imperfect quantum channel reduces the range of state $\left|\psi_{\text {in }}\right\rangle$ that is accurately teleported [1]. Figure 2 shows the fidelity $F(\theta, \phi)$ for various types of noises at $4 \kappa \tau=3.0$. For the noisy channel defined by $L_{2, z}$ and $L_{3, z}$, the fidelity $F(\theta, \phi)$ is always the maximum value of 1 at $\theta=0, \pi$, irrespective of $\kappa \tau$ as depicted in Fig. 2(a). These angles indicate states $|0\rangle$ and $|1\rangle$, which are eigenstates of $\sigma_{z}$. From $F(\theta, \phi) \simeq \frac{1}{2}(1$ $+\cos ^{2} \theta$ ) in the limit of $4 \kappa \tau \gg 1$, the range of states to be teleported with fidelity $F \geqslant 3 / 4$ is determined by $0 \leqslant \theta \leqslant \pi / 4$ and $3 \pi / 4 \leqslant \theta \leqslant \pi$. The teleported states with fidelity $2 / 3$ are in the region determined by $\cos \theta \geqslant 1 / \sqrt{3}$ or $\cos \theta \leqslant-1 / \sqrt{3}$. When $L_{2, x}$ and $L_{3, x}$ are applied to qubits 2 and 3 , we get $F(\theta, \phi)=1$ at $\theta=\pi / 2$ and $\phi=0, \pi$ for $4 \kappa \tau \gg 1$, which shown in Fig. 2(b). These angles represent states $|\psi\rangle$ $=(1 / \sqrt{2})(|0\rangle+|1\rangle)$ and $|\psi\rangle=(1 / \sqrt{2})(|0\rangle-|1\rangle)$, i.e., eigen- 
states of $\sigma_{x}$. The range of states accurately teleported is depicted by contours in Figs. 2(a) and 2(b).

When the quantum channel is subject to noise in one direction, we obtain the average fidelity as depicted in Fig. 3 (denoted by case $B-1$ )

$$
F_{\text {av }}(\tau)=\frac{2}{3}+\frac{1}{3} e^{-4 \kappa \tau} .
$$

The average fidelity decays exponentially to the limiting value of $2 / 3$. This is the best possible score when Alice and Bob communicate with each other only through the classical channel $[3,5]$.

Consider the case where the quantum channel is affected by isotropic noise, which is described by six Lindblad operators, $L_{2, \alpha}$ and $L_{3, \alpha}$ with $\alpha=x, y, z$. Then the analytic calculation of the fidelity can be written by

$$
F_{\mathrm{av}}=F(\theta, \phi)=\frac{1}{2}+\frac{1}{2} e^{-8 \kappa \tau} .
$$

As depicted in Fig. 2(c), the fidelity $F(\theta, \phi)$ is independent of angles of input states, $\theta$ and $\phi$, for any value of $\kappa \tau$. For the quantum channel subject to isotropic noise, one could not find the range of states that is accurately teleported. As shown in Fig. 3 (case $B$-2), the average fidelity decays to the value of $1 / 2$. The number $1 / 2$ can be obtained when Alice and Bob cannot communicate at all and Bob merely selects a state at random.

It should be noted that except decoherence rates $\kappa \tau$, the overall features of cases $A$ and $B$ are identical. This implies that if a state to be teleported is realized by a single particle and not by an ensemble, one may not be able to identify whether the state to be teleported is mixed or the quantum channel is noisy.

Cases $C$ and D: Noise during Bell's measurement or the unitary operation. When Alice performs the Bell's measurement or Bob does the unitary operation on his particle of an entangled pair, noise may take place as depicted by the boxes $C$ or $D$ in Fig. 1. In contrast to cases $A$ and $B$, it seems to be difficult to find analytic solutions of Eq. (1) for cases $C$ and $D$ because of the time dependence of the qubit Hamiltonian $H_{S}(t)$. Alice's Bell measurement on qubits 1 and 2 could be done by a controlled-NOT gate (CNOT) on qubits 1 and 2, and a Hadamard gate $H_{1}$ on qubit 1 as shown in Fig. 1. With a qubit system modeled by Hamiltonian, Eq. (2), the CNOT gate acting on qubits 1 and 2 could be implemented by the pulse sequence $[12,13] e^{-i \pi / 4} \mathrm{H}_{1} R_{2 x}(\pi / 2) R_{1 x}$ $(-\pi / 2) U_{2 b}^{12}(\pi / 4) R_{1 x}(\pi) U_{2 b}^{12}(\pi / 4) H_{1}$. Here $R_{j x}(\theta)$ $\equiv e^{i \sigma_{x}^{(j)} \theta / 2}$ is a rotation of qubit $j$ by angle $\theta$ about the $x$ axis. A two-qubit operation $U_{2 b}^{12}(\theta)$ on qubits 1 and 2 is implemented by turning on the coupling $J_{12}$ for a time $t$ corresponding to $\theta \equiv J_{12} t / \hbar$. During each qubit operation, the noise modeled by Lindblad operators is also switched on. Thus, it does not seem to be simple to obtain an analytic solution and we take a numerical method to solve the problem.

Consider the noise modeled by the Lindblad operators $L_{1 z}$ and $L_{2 z}$ for case $C$ and $L_{3 z}$ for case $D$. Here the noise is (a) Case C

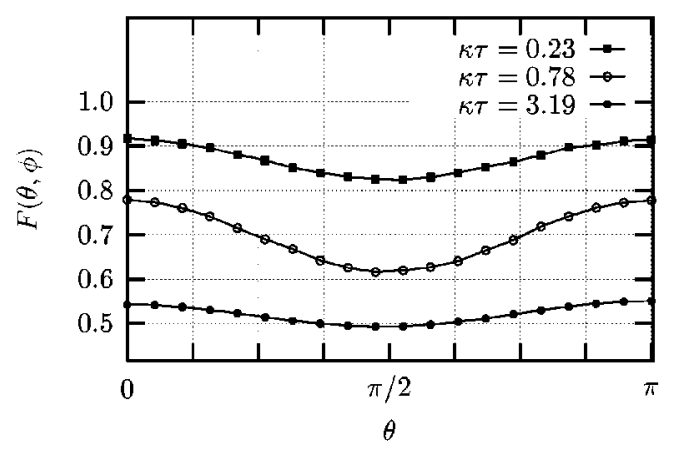

(b) Case D

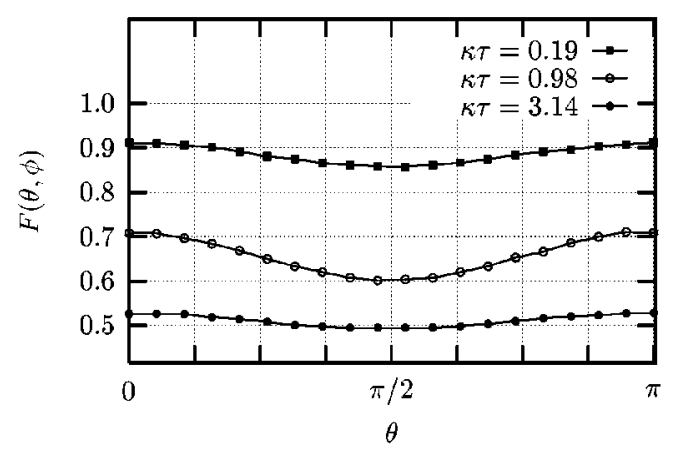

(c) $g(\kappa \tau) \equiv \max \{F(\theta, \phi)\}-\min \{F(\theta, \phi)\}$

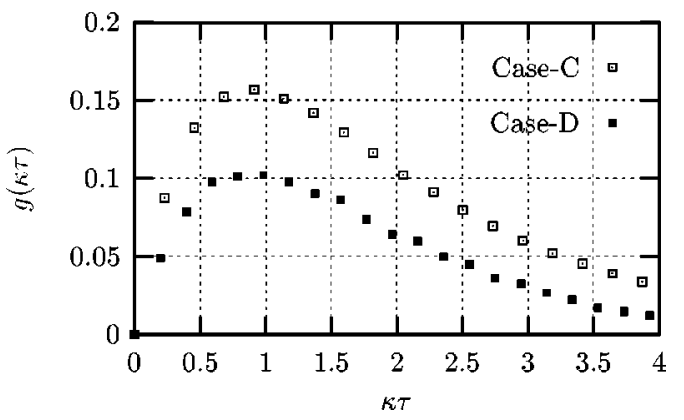

FIG. 4. Fidelity $F(\theta, \phi)$ vs angle $\theta$ for various values of $\kappa \tau$. (a) For case $C$, noise is modeled by $L_{1 z}$ and $L_{2 z}$ and (b) for case $D$ by $L_{3 z} . F(\theta, \phi)$ is independent of angle $\phi$ because of the cylindrical symmetry of $L_{i z}$ with $i=1,2,3$. Differences between the maximum and minimum values of the fidelity $F(\theta, \phi)$ are plotted as a function of $\kappa \tau$.

switched on during the time interval $\tau$ corresponding to the total operation time that it takes to implement Bell's measurement or controlled $X$ and $Z$ operations. The time interval $\tau$ depends on the operation times of a single gate or a twoqubit gate, proportional to $h /\left|\mathbf{B}^{(i)}\right|$ and $h / J_{i j}$, respectively. Figure 4 shows the fidelity $F(\theta, \phi)$ as a function of angle $\theta$ for various values of $\kappa \tau$. In contrast to the previous cases (cases $A-1, A-2, B-1$, and $B-2$ ) whose fidelity is given by Eq. (9) or (10), in cases $C$ and $D$ the degrees of the dependence of fidelity $F(\theta, \phi)$ on angles $\theta$ is maximum at a certain value of $\kappa \tau$. Figure 4(c) shows the differences between the maximum and minimum values of the fidelity, $g(\kappa \tau)$ $\equiv \max \{F(\theta, \phi)\}-\min \{F(\theta, \phi)\}$. It is not clear why $g(\kappa \tau)$ has the maximum at $\kappa \tau \approx 0.98$. As depicted in Fig. 5, the average 


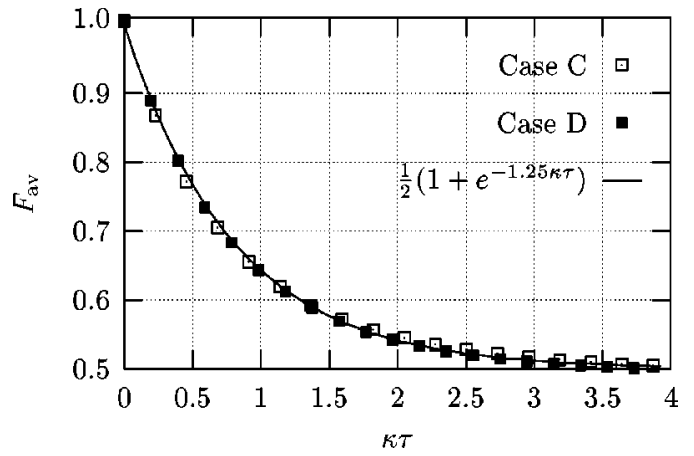

FIG. 5. Average fidelity $F_{\text {av }}$ as a function of $\kappa \tau$ for cases $C$ (dotted boxes) and $D$ (filled boxes).

fidelity falls to the value of $1 / 2$ and is approximately fitted by

$$
F_{\mathrm{av}}(\tau)=\frac{1}{2}+\frac{1}{2} e^{-1.25 \kappa \tau}
$$

One sees that cases $A$ and $B$ entirely differ from cases $C$ and $D$. Although an analytic solution for case $C$ and $D$ cannot be obtained, it can be understood why the average fidelity decays to $1 / 2$ despite noise described by the Lindblad operator acting in one direction. Consider a rotation of a qubit about the $x$ axis in the presence of noise modeled by $L_{z}$. A simple calculation shows that the Bloch vector $\mathbf{r}$ of a qubit, which is defined by $\rho=\frac{1}{2}(1+\mathbf{r} \cdot \boldsymbol{\sigma})$, falls to zero for any initial state. This means the qubit is depolarized and becomes a totally mixed state. Thus the average fidelity decays to $1 / 2$ when a gate operation is done in the presence of noise.

It is valuable to discuss our results in connection with the previous studies [3]. In Ref. [3], Popescu illustrated an ex- ample of a mixed pair that does not violate any Bell inequality but has an average fidelity $3 / 4$ for arbitrary input states, given by $\rho=\frac{1}{8} I+\frac{1}{2}\left|\Psi^{-}\right\rangle\left\langle\Psi^{-}\right|$with $\left|\Psi^{-}\right\rangle=(1 / \sqrt{2})(|01\rangle$ $-|10\rangle)$. Our calculation in which the quantum channel is described by this mixed state shows the fidelity $F(\theta, \phi)$ $=3 / 4$, independent of angles $\theta$ and $\phi$, and thus gives us an average fidelity $F_{\mathrm{av}}=3 / 4$. Horodecki et al. [6] showed that the optimal fidelity of the standard quantum teleportation is given by $f=\left(2 F_{A B}+1\right) / 3$, where $F_{A B}$ is the singlet fraction of the quantum channel. From Eq. (14), one can write $F_{A B}$ $=\left(1+e^{-4 \kappa \tau}\right) / 2$.

In conclusion, we calculated the fidelity and the average fidelity of quantum teleportation subject to various types of noises during different steps of the teleportation. We examined the range of states that can be accurately teleported. Among states to be teleported, the eigenstate of the Lindblad operators is less sensitive to the noise. It was shown that one cannot distinguish whether an unknown state to be teleported, which is realized by a single particle, is mixed or the quantum channel is noisy. We found the dependence of the average fidelity on the type of noise affecting the quantum channel. If the quantum channel is subject to isotropic noise, the average fidelity may decay to $1 / 2$. On the other hand, if the noisy quantum channel is described by a single Lindblad operator, the average fidelity is always greater than the value of $2 / 3$, the best possible value that can be obtained only by the classical communication.

We thank Dr. Jaewan Kim for helpful discussions. This work was supported by the Brain Korea 21 Project of the Korea Ministry of Education, the NRL program of the Korean Ministry of Science and Technology, and KOSEF via eSSC at POSTECH.
[1] C.H. Bennett, G. Brassard, C. Crépeau, R. Jozsa, A. Peres, and W.K. Wootters, Phys. Rev. Lett. 70, 1895 (1993).

[2] D. Bouwmeester, J.-W. Pan, K. Mattle, M. Eibl, H. Weinfurter, and A. Zeilinger, Nature (London) 390, 575 (1997); D. Boschi, S. Branca, F. De Martini, L. Hardy, and S. Popescu, Phys. Rev. Lett. 80, 1121 (1998).

[3] S. Popescu, Phys. Rev. Lett. 72, 797 (1994).

[4] R. Horodecki, M. Horodecki, and P. Horodecki, Phys. Lett. A 222, 21 (1996).

[5] S. Massar and S. Popescu, Phys. Rev. Lett. 74, 1259 (1995).

[6] M. Horodecki, P. Horodecki, and R. Horodecki, Phys. Rev. A 60, 1888 (1999).
[7] K. Banaszek, Phys. Rev. Lett. 86, 1366 (2001).

[8] S. Ishizaka, Phys. Rev. A 63, 034301 (2001).

[9] M. A. Nielsen and I. L. Chuang, Quantum Computation and Quantum Information (Cambridge University Press, Cambridge, England, 2000).

[10] G. Lindblad, Lett. Math. Phys. 48, 119 (1976).

[11] R. Alicki and K. Lendi, Quantum Dynamical Semigroups and Applications, Lecture Notes in Physics Vol. 286 (SpringerVerlag, Berlin, 1987).

[12] Y. Makhlin, G. Schön, and A. Shnirman, Rev. Mod. Phys. 73, 357 (2001).

[13] S. Oh, Phys. Rev. B 65, 144526 (2002). 